\title{
On the semi-dynamical reflection equation: solutions and structure matrices
}

\author{
J. Avan! 1 and C. Zambon² \\ Laboratoire de Physique Théorique et Modélisation \\ Université de Cergy-Pontoise (CNRS UMR 8089), Saint-Martin 2 \\ 2 avenue Adolphe Chauvin, 95302 Cergy-Pontoise Cedex, France
}

\begin{abstract}
Explicit solutions of the non-constant semi-dynamical reflection equation are constructed, together with suitable parametrizations of their structure matrices. Considering the semi-dynamical reflection equation with rational non-constant Arutyunov-Chekhov-Frolov structure matrices, and a specific meromorphic ansatz, it is found that only two sets of the previously found constant solutions are extendible to the non-constant case. In order to simplify future constructions of spin-chain Hamiltonians, a parametrization procedure is applied explicitly to all elements of the semi-dynamical reflection equation available. Interesting expressions for 'twists' and $R$ matrices entering the parametrization procedure are found. In particular, some expressions for the $R$-matrices seem to appear here for the first time. In addition, a new set of consistent structure matrices for the semi-dynamical reflection equation is obtained.
\end{abstract}

\footnotetext{
${ }^{1}$ E-mail: avan@u-cergy.fr

${ }^{2}$ E-mail: cristina.zambon@u-cergy.fr
} 


\section{Introduction}

Dynamical extensions of Sklyanin-type quantum reflection algebras [1], [2], [3] have been introduced and quite extensively studied in the last years [4], [5]. The so called semi-dynamical reflection algebra, exemplified in [4], was formulated generically in [5] and later used as a basic algebraic structure to yield formal spin-chain quantum integrable Hamiltonians [6]. Generic consistent parametrizations of its matrices were then proposed in [7], leading to remarkably simplified factorized formulas for the generating monodromy matrices. Together with the classification of scalar (non-operatorial) solutions $K$ to the specific semi-dynamical reflection equation (SDRE), started in [8] for the constant case, this procedure is expected to lead to new, fully explicit, spin-chain Ruijsenaar-Schneider (RS) type Hamiltonians. The results presented in this article represent another step in this direction. Explicit resolutions of the parametrization described in [7], together with the subsequent partial classifications of non-constant scalar $K$ solutions will be given. Indeed, the construction of tractable (i.e. locally interacting) spinchain type Hamiltonians, contrary to the pure $N$-body system Hamiltonians, requires a priori the consideration of non-constant, spectral parameter dependent solutions [1]. In this context, the parametrization proposed in [7] considerably simplifies the form of the relevant monodromy matrices, and therefore it is a key ingredient to explicitly build the Hamiltonians.

The SDRE is a quadratic constraint equation for generators of a quantum algebra $\mathcal{G}$ encapsulated into the matrix $K$. Such a constraint is represented as an equation in $\operatorname{End}(\mathcal{U}) \otimes \operatorname{End}(\mathcal{U})$ where $\mathcal{U}$ is a given vector space known as the auxiliary space. This space can be a finite dimensional space $V$ or a loop space $V \otimes \mathbb{C}[u]$. The general form of the SDRE considered in this article is:

$$
A_{12}(u, v ; \lambda) K_{1}(u ; \lambda) B_{12}(v ; \lambda) K_{2}\left(v ; \lambda+\gamma h_{1}\right)=K_{2}(v ; \lambda) C_{12}(u ; \lambda) K_{1}\left(u ; \lambda+\gamma h_{2}\right) D_{12}(u-v ; \lambda),
$$

where $A, B, C$ and $D$ are $\mathbb{C}$-number matrices known as structure matrices. All elements appearing in (1.1) depend on a set of complex variables, collectively denoted $\lambda \equiv\left\{\lambda_{i}, i=\right.$ $1, \ldots, n\}$ and known as dynamical variables. If the auxiliary space is a loop space, there is also a spectral parameter dependence in $A, B, C, D, K$ represented by the complex variables $u$ and $v$. Notice that the matrix $D$ may have a more general dependence in the spectral parameters, like $A$, but this leads to subsequent difficulties when deriving the commuting Hamiltonians [7]. The dynamical variables $\left\{\lambda_{i}\right\}$ are interpreted as coordinates on the dual $\mathbf{h}^{*}$ of a $n$-dimensional abelian subalgebra $\mathbf{h}$ of a simple Lie algebra $\mathbf{g}$.

Given a basis $h \equiv\left\{h^{i}, i=1, \ldots, n\right\}$ of $\mathbf{h}^{*}$ (with $\left\{h_{i}, i=1, \ldots, n\right\}$ basis in $\mathbf{h}$ ), and setting $\lambda=\sum_{i=1}^{n} \lambda_{i} h^{i}$, it is possible to define formally

$$
f(\lambda+\gamma h) \equiv e^{\gamma \mathcal{D}} f(\lambda) e^{-\gamma \mathcal{D}}, \quad \mathcal{D}=\sum_{i=1}^{n} h_{i} \partial_{\lambda_{i}},
$$

where $f(\lambda)$ is a differentiable function on $\mathbf{h}^{*}$ and the auxiliary space $\mathcal{U}$ is assumed to be a diagonalizable irreducible module of $\mathbf{h}$. In order to simplify the notation, it will be often set $f(h) \equiv f(\lambda+\gamma h)$.

The structure matrices are supposed to satisfy the following zero-weight conditions

$$
\left[h_{i} \otimes \mathbb{1}, B_{12}\right]=0, \quad\left[\mathbb{1} \otimes h_{i}, C_{12}\right]=0, \quad\left[h_{i} \otimes \mathbb{1}+\mathbb{1} \otimes h_{i}, D_{12}\right]=0, \quad i=1, \ldots, n .
$$


Moreover, the assumed associativity of the semi-dynamical reflection algebra $\mathcal{G}$ yields, as sufficient consistency conditions, that the structure matrices obey a set of YB-like equations [5]. Such equations have been reformulated in [7] to take into account certain freedom enjoyed by the structure matrices due to the form-invariance of the SDRE (1.1) under suitable transformations. In fact, multiplying on the left hand side equation (1.1) by $(g \otimes \mathbb{1})$, where $g$ is an automorphism of the auxiliary space $\mathcal{U}$, leads to an equivalent - though with a different definition of structure matrices - formulation of the exchange relations satisfied by the generators of the algebra $\mathcal{G}$, which are encapsulated into the unmodified matrix $K$. Taking into account this property, the YB-like consistency equations can be written as follows

$$
\begin{array}{ll}
\text { a } & A_{12} A_{13}{ }^{g g} A_{23}=A_{23}{ }^{g g} A_{13} A_{12}{ }^{g g}, \\
\text { b } & A_{12} C_{13}{ }^{g} C_{23}=C_{23}{ }^{g 2} C_{13} A_{12}\left(h_{3}\right)^{g g}, \\
\text { c } & D_{12} B_{13} B_{23}\left(h_{1}\right)^{g 3}=B_{23} B_{13}\left(h_{2}\right)^{g 3} D_{12}, \\
\text { d } & D_{12}\left(h_{3}\right) D_{13} D_{23}\left(h_{1}\right)=D_{23} D_{13}\left(h_{2}\right) D_{12},
\end{array}
$$

where

$$
M_{12}{ }^{g g} \equiv g_{1} g_{2} M_{12} g_{1}^{-1} g_{2}^{-1}, \quad M_{12}^{g_{1}} \equiv g_{1} M_{12} g_{1}^{-1}, \quad M_{12}^{g_{2}} \equiv g_{2} M_{12} g_{2}^{-1},
$$

and $g$ is the automorphism of the auxiliary space. Note that (1.4-d) is the Gervais-NeveuFelder (GNF) equation [9], and it is unmodified by this extension. In addition, for consistency conditions, $B_{12}=C_{21}$.

In this article only the sets of matrices $A, B, C, D$ for which there exists scalar invertible solutions $K$ to (1.1) will be taken into account. At present, only one of these sets is known, namely the Arutyunov-Chekhov-Frolov (ACF) solution [4], which is associated to the RS models based on the Lie algebra data $g l(n)[10$. In this case the structure matrices obey the generalized YB set of equations (1.4) where the automorphism $g$ represents a shift in the spectral parameter $u$ as $g=\exp \left(-\gamma \frac{d}{d u}\right)$. In the limit of non-spectral parameter dependence the automorphism reduces to $g=\mathbb{1}$. For this set of matrices $A, B, C, D$, the auxiliary space $\mathcal{U}$ is a finite dimensional loop space $V \otimes \mathbb{C}[u]$ with $V=\mathbb{C}^{n}$ and $\mathbf{h}$ is the Cartan subalgebra of the Lie algebra $g l(n)$. As a consequence, for the matrix $K$ in (1.1), the definition (1.2) translates into

$$
K_{2}\left(h_{1}\right) \equiv \sum_{j=1}^{n} h_{j} \otimes K\left(\lambda_{j}+\gamma\right)
$$

Given a basis $\left\{e_{i}, i=1, \ldots, n\right\}$ of $V, e_{i j}=e_{i} \otimes e_{j}$ (with $e_{j j} \equiv h_{j}$ ) represents the usual matrix basis and the rational ACF structure matrices read [4]

$$
\begin{aligned}
A_{12}(u, v ; \lambda) & =A_{12}^{\infty}(\lambda)+\frac{\gamma}{(u-v)} d_{12}+\frac{\gamma}{v} b_{12}-\frac{\gamma}{(u+\gamma)} b_{21}, \\
B_{12}(v ; \lambda) & =B_{12}^{\infty}(\lambda)-\frac{\gamma}{(v+\gamma)} b_{12}, \quad C_{12}(u ; \lambda)=B_{21}(v ; \lambda), \\
D_{12}(u-v ; \lambda) & =D_{12}^{\infty}(\lambda)+\frac{\gamma}{(u-v)} d_{12},
\end{aligned}
$$


with

$$
\begin{aligned}
& A_{12}^{\infty}(\lambda)=\mathbb{1} \otimes \mathbb{1}+\sum_{i \neq j=1}^{n} x_{i j}\left(e_{i i}-e_{i j}\right) \otimes\left(e_{j j}-e_{j i}\right), \\
& B_{12}^{\infty}(\lambda)=\mathbb{1} \otimes \mathbb{1}+\sum_{i \neq j=1}^{n} y_{i j} e_{j j} \otimes\left(e_{i i}-e_{i j}\right) ; \quad C_{12}^{\infty}(\lambda)=B_{21}^{\infty}(\lambda), \\
& D_{12}^{\infty}(\lambda)=\mathbb{1} \otimes \mathbb{1}+\sum_{i \neq j=1}^{n} x_{i j}\left(e_{i j} \otimes e_{j i}-e_{i i} \otimes e_{j j}\right),
\end{aligned}
$$

and

$$
\begin{aligned}
& d_{12}=\sum_{i, j=1}^{n} e_{i j} \otimes e_{j i}, \quad b_{12}=\sum_{i, j=1}^{n} e_{i i} \otimes e_{j i}, \quad c_{12} \equiv b_{21}, \\
& x_{i j}=\frac{\gamma}{\left(\lambda_{i}-\lambda_{j}\right)} \equiv \frac{\gamma}{\lambda_{i j}}, \quad y_{i j}=\frac{\gamma}{\left(\lambda_{i}-\lambda_{j}-\gamma\right)} \equiv \frac{\gamma}{\left(\lambda_{i j}-\gamma\right)} .
\end{aligned}
$$

The matrices (1.10-1.12) will be denoted as rational 'constant' ACF matrices because they do not depend on the spectral parameter. Similarly, matrices (1.7-1.9) will be called non-constant ACF matrices. They exhibit spectral parameter dependence and reduce to the previous set $(1.10-1.12)$ in the limit $u, v,(u-v) \longrightarrow \infty$.

The parametrization procedure proposed in [7] makes use of quantum group-like objects such as $R$-matrices and Drinfeld's twists for building the $A, B, C, D$ and $K$ matrices. It allows to simplify significantly the expressions for the monodromy matrices found previously [6], and therefore to facilitate the explicit construction of integrable spin-chain Hamiltonians.

The purpose of the present article is to provide explicit realizations of the parametrization procedure proposed in [7] making use of the specific matrices $D$ available in (1.9) and (1.12), and to classify, at least partially, non-constant solutions $K$ of the SDRE. The first part of the article is focused on the search of solutions of equation (1.1) using the structure matrices (1.7) 1.9). The final aim is to extend the results obtained in [8] for the rational constant structure matrices (1.10,1.12) to the case with spectral parameter dependence. The second part of the paper will be devoted to the parametrization procedure for which three distinct situations will be considered. Making use of the ACF set of structure matrices, the parametrization procedure will be applied first to the simpler case $g=\mathbb{1}$ and later to the more complicated situation in which $g=\exp \left(-\gamma \frac{d}{d u}\right)$. Finally, adopting an alternative parametrization for matrices $D$ in (1.12) and (1.9), which is provided in [11], it will be shown how the parametrization procedure leads to an alternative set of solutions of equations (1.4), namely to new structure matrices $A$, $B, C$. Full analysis of the SDRE built by these matrices will be left to further studies, even if some information concerning the solutions of this equation can be deduced making use of the parametrization procedure. It should be emphasized that the existence of this new set of structure matrices relies on the availability of distinct de-twisting procedures for a single $D$ matrix. In fact, from [7] a set of consistent structure matrices $A, B, C, D$ is provided as soon as a $D$-matrix is chosen and a particular cocycle twist-like formulation of $D$ is specified. Further remarks concerning this point will be added later. 


\section{Solutions of the non constant rational SDRE}

In this section, making use of the rational ACF set of solutions for the equations (1.4), the problem to discover and classify the matrices $K$ solving the SDRE will be addressed. In [8], as it was pointed out before, this problem has been already tackled in the case of no spectral parameter dependence. Four sets of solutions were identified, namely

$$
\begin{array}{rlrl}
\text { Ia } & K_{i j}^{\infty}(\lambda) & =\frac{f+\lambda_{i}}{f+\lambda_{j}} \prod_{a \neq j}^{n} \frac{\gamma}{\lambda_{j a}}, \\
\text { IIa } & K_{i j}^{\infty}(\lambda)=\left(f-\Lambda_{i j}\right) \prod_{a \neq j}^{n} \frac{\gamma}{\lambda_{j a}}, \quad \Lambda_{i j}=\sum_{a=1}^{n} \lambda_{a}-\left(\lambda_{i}+\lambda_{j}\right) \\
\text { Ib } & K_{i j}^{\infty}(\lambda)=\frac{f+\Lambda_{j}}{f+\Lambda_{i}} \prod_{a \neq j}^{n} \frac{\gamma}{\lambda_{j a}}, \quad \Lambda_{i}=\sum_{a=1}^{n} \lambda_{a}-\left(\lambda_{i}\right) \\
\text { IIb } & K_{i j}^{\infty}(\lambda)=\frac{f}{f-\lambda_{i j}} \prod_{a \neq j}^{n}\left(1+\frac{f}{\lambda_{j a}}\right),
\end{array}
$$

where $f$ is a function $\gamma$-periodic on each dynamical variable. All other solutions can be obtained from these sets by multiplying the $K$-matrix on the right by a diagonal matrix $N_{i i}(\lambda)$ satisfying the following flatness condition

$$
N_{i i}(\lambda) N_{j j}\left(\lambda+\gamma h_{i}\right)=N_{j j}(\lambda) N_{i i}\left(\lambda+\gamma h_{j}\right)
$$

In particular, the matrix $N_{j j}=0, N_{i i}=1, i \neq j$ allows to obtain a matrix $K$ with the entries $K_{k j}=0, k=1, \ldots, n$. In this case, the periodicity condition on the dynamical variable $\lambda_{j}$ for the function $f$ is omitted. It can be noticed that for $n=2$ solutions (2.1-Ia) and (2.1-IIa) collapse to $(2.1-\mathbf{I b})$ and (2.1-IIb), respectively. Moreover, the only invertible matrices are represented by solutions (2.1-IIb), since matrices (2.1 -Ia) and (2.1-Ib) have rank 1 while matrices (2.1 -IIa) have rank 2. Finally, note that in the limit $f \longrightarrow 0$ solutions (2.1-IIb) reduce to the trivial solution $\mathbb{1}$.

The classification of the matrices $K$ solving the SDRE will now be extended to the case with spectral parameter dependence. In order to simplify the notation, the explicit dependence on the dynamical variables will be omitted in what follows. Because of the particular form of the structure matrices, and without making any assumption on the unknown matrices $K$, the SDRE (1.1) can be rewritten in a more appealing form as

$$
\begin{aligned}
& \left(A_{12}(u, v)-\frac{\gamma}{(u-v)} d_{12}\right) K_{1}(u) B_{12}(v) K_{2}\left(v ; h_{1}\right) \\
= & \left(K_{2}(v) C_{12}(u) K_{1}\left(u ; h_{2}\right)-K_{2}(u) C_{12}(v) K_{1}\left(v ; h_{2}\right)\right) \frac{\gamma}{(u-v)} d_{12} \\
& +K_{2}(v) C_{12}(u) K_{1}\left(u ; h_{2}\right) D_{12}^{\infty} .
\end{aligned}
$$

The advantage of this formulation is to gather together in a more compact way the terms 
proportional to the factor $1 /(u-v)$. Then, the following ansatz for the matrix $K$ will be used

$$
K(u, \lambda)=\sum_{l=0}^{N}\left(\frac{\gamma}{u}\right)^{l} k^{(l)}(\lambda), \quad k^{(0)}(\lambda) \equiv K^{\infty}(\lambda) .
$$

This expansion in powers of $1 / u$ represents a natural extension, as rational function, of the solutions (2.1) to which it reduces in the limit $u \longrightarrow \infty$. Note that in (2.4) the location of the poles at $u=0$ is just a matter of choice. Multiplying such an ansatz by

$$
\prod_{l=1}^{N}\left(\frac{u}{u-u_{0}}\right)^{l}
$$

allows to obtain an equivalent ansatz with the poles shifted at $u=u_{0}$. Reciprocally, given any matrix $K$ with a finite set of poles, it can always be brought back to the form (2.4) by a suitable multiplicative factor like (2.5).

Once (2.4) is plugged into the expression (2.3), it is noticed that all terms coming from the second line, and proportional to $1 /(u-v)$ can be combined together in such a way to eliminate completely this factor. For instance:

$$
\frac{1}{(u-v)}\left[\frac{1}{v(u+\gamma)}-\frac{1}{u(v+\gamma)}\right]=\frac{\gamma}{u v(u+\gamma)(v+\gamma)}
$$

Then, using the property

$$
c_{12} k_{1}^{(l)} b_{12} k_{2}^{(l)}\left(h_{1}\right)=k_{2}^{(l)} c_{12} k_{1}^{(l)}\left(h_{2}\right) d_{12}, \quad l=0,1, \ldots, N
$$

some simplifications can be performed amongst terms coming from the first and the second lines of (2.3) . The remaining terms must be treated with care. First of all, the powers of the spectral parameters appearing in each term must be reduced as much as possible by decomposition in prime elements. Finally, making use of the property

$$
\left(A^{\infty}-b\right)_{12} k_{1}^{(l)} b_{12} k_{2}^{(l)}\left(h_{1}\right)=0, \quad l=0,1, \ldots, N
$$

and the reduction explained above, further simplifications are possible. The expression obtained splits into several relations, each of them gathering algebraically independent terms. They represents constraints for the elements of the matrices $k^{(l)}$, which must be analyzed carefully by projecting them onto the matrix elements $\left(e_{i j} \otimes e_{k l}\right) i, j, k, l=1, \ldots, n$.

Starting with the simplest ansatz for the matrices $K$, namely the expression (2.4) with $l=1$, the expression (2.3) translates into 8 relations, namely 


$$
\begin{aligned}
& A_{12}^{\infty} k_{1}^{(0)} B_{12}^{\infty} k_{2}^{(0)}\left(h_{1}\right)=k_{2}^{(0)} C_{12}^{\infty} k_{1}^{(0)}\left(h_{2}\right) D_{12}^{\infty} \\
& b_{12} k_{1}^{(0)}\left(B^{\infty}-b\right)_{12} k_{2}^{(1)}\left(h_{1}\right)=0 \\
& b_{12} k_{1}^{(1)}\left(B^{\infty}-b\right)_{12} k_{2}^{(1)}\left(h_{1}\right)=0, \\
& \left(A^{\infty}-c\right)_{12} k_{1}^{(1)} B_{12}^{\infty} k_{2}^{(1)}\left(h_{1}\right)-k_{2}^{(1)}\left(C^{\infty}-c\right)_{12} k_{1}^{(1)}\left(h_{2}\right) D_{12}^{\infty} \\
& +k_{2}^{(0)}\left(C^{\infty}-c\right)_{12} k_{1}^{(1)}\left(h_{2}\right) d_{12}+(b-d)_{12} k_{1}^{(1)}\left(B^{\infty}-b\right)_{12} k_{2}^{(0)}\left(h_{1}\right)=0 \text {, } \\
& \left(A^{\infty}-c\right)_{12} k_{1}^{(1)} B_{12}^{\infty} k_{2}^{(0)}\left(h_{1}\right)-k_{2}^{(0)}\left(C^{\infty}-c\right)_{12} k_{1}^{(1)}\left(h_{2}\right) D_{12}^{\infty}=0 \text {, } \\
& \text { a } \quad c_{12}\left(k^{(1)}-k^{(0)}\right)_{1} B_{12}^{\infty} k_{2}^{(0)}\left(h_{1}\right)-k_{2}^{(0)} c_{12}\left(k^{(1)}-k^{(0)}\right)_{1}\left(h_{2}\right) D_{12}^{\infty}=0, \\
& \text { b } \quad c_{12}\left(k^{(1)}-k^{(0)}\right)_{1}\left(B_{12}^{\infty}-b\right)_{12} k_{2}^{(1)}\left(h_{1}\right)-k_{2}^{(1)} c_{12}\left(k^{(1)}-k^{(0)}\right)_{1}\left(h_{2}\right)\left(D_{12}^{\infty}-d\right)_{12}=0 \text {, } \\
& \text { c } \quad A_{12}^{\infty} k_{1}^{(0)} B_{12}^{\infty} k_{2}^{(1)}\left(h_{1}\right)-k_{2}^{(1)} C_{12}^{\infty} k_{1}^{(0)}\left(h_{2}\right) D_{12}^{\infty}+b_{12} k_{1}^{(0)}\left(B^{\infty}-b\right)_{12} k_{2}^{(0)}\left(h_{1}\right)=0 .
\end{aligned}
$$

Equation (2.9), which involves only the matrix $k^{(0)}$, has already been investigated and its solutions have been listed in (2.1) $\left(k^{(0)} \equiv K^{\infty}\right)$. All other 8 relations incorporate both $k^{(0)}$ and $k^{(1)}$ matrices. When they are analyzed, one discovers that (2.10) represents a strong constraint for the matrix $k^{(1)}$.

Consider first the case when $k^{(0)}$ has no zero entry, then expression (2.10) states the following

$$
k_{i j}^{(1)}=k_{k j}^{(1)}, \quad i \neq k=1, \ldots, n .
$$

As a consequence the relations (2.11) become identities and only the 3 relations (2.12) remain to be investigated. For instance, $(2.12 \mathrm{r}$ a) allows to establish whether the sets of solutions listed in (2.1) can be extended or not, and to specify the form of the extensions. Provided $k^{(1)}$ has no zero entry, it turns out that (2.1-Ia) and (2.1-IIa) are not extendable to a first order solution $k^{(1)}$. On the contrary, the solutions $(2.1-\mathbf{I b})$ and $(2.1-\mathbf{I I b})$ can be uniquely extended as follows

$$
\text { Ib } \begin{aligned}
K_{i j}(u ; \lambda) & =\left(f+\Lambda_{j}\right)\left(\frac{1}{f+\Lambda_{i}}-\frac{1}{u}\right) \prod_{a \neq j}\left(\frac{\gamma}{\lambda_{j a}}\right), \quad \Lambda_{i}=\sum_{a=1}^{n} \lambda_{a}-\left(\lambda_{i}\right), \\
\text { IIb } \quad K_{i j}(u ; \lambda) & =f\left(\frac{1}{f-\lambda_{i j}}-\frac{1}{u}\right) \prod_{a \neq j}\left(\frac{f}{\lambda_{j a}}+1\right) .
\end{aligned}
$$

The constraints provided by expressions $(2.12-\mathbf{b})$ and $(2.12-\mathbf{c})$ are automatically satisfied by the sets of solutions (2.14). Notice that solution (2.14-IIb) with constant $f$ coincides with the solution found by ACF in [4]. Moreover, it can be shown that allowing a column in the matrix $k^{(1)}$ to be zero, constraints (2.12) force all elements of the corresponding column in $k^{(0)}$ to be identical. However, this can only happen provided these elements are equal to zero, which is not allowed by the starting hypothesis. Then, the case $k^{(0)}$ with no zero entry is completely covered.

Consider now the case when $k^{(0)}$ has a zero entry, and therefore the whole column is zero, as established in [8]. Solutions $K^{\infty}=k^{(0)}$ with one or more zero-columns can be obtained by 
performing suitable simple transformations on the full solutions (2.1), and this then builds the whole set of constant solutions. Here similar results can be established. Assuming $k^{(0)}$ has a column set to zero, the corresponding column in $k^{(1)}$ is forced to be zero as well. Therefore, it is possible to conclude that solutions $K$ with columns set to zero are possible, and they are obtained by setting to zero one or several columns of solutions (2.14), since the specific form of non-zero columns does not depend on the existence of other zero-columns. Finally, it can be noticed that the limit $f \longrightarrow 0$ in $(2.14-\mathbf{I I b})$ provides again the solution $\mathbb{1}$ where the spectral parameter does not appear. However, such a solution can be extended in a way to include a spectral parameter dependence as follows

$$
K(u ; \lambda)=\mathbb{1}\left(1+\frac{f^{\prime}}{u}\right)
$$

where $f^{\prime}$ is any function $\gamma$-periodic on each dynamical variable.

Attempts to find alternative solutions by truncating the expansion (2.4) to orders higher than $l=1$ proved to be unsuccessful. First of all, it is possible to show that to the order $l=2$ the ansatz (2.4) with $k^{(0)}$ and $k^{(1)}$ given by (2.14) is not a solution of the set of equations coming from expression (2.3), unless $k^{(2)}=0$. Therefore, the only way out is to reconsider the situation with both matrices $k^{(1)}$ and $k^{(2)}$ unknown. In fact, the relation (2.9) is the only constraint which emerged unaltered by using the ansatz (2.4) for a generic order $l$. All other relations stemming from (2.3) depend on the order of the ansatz (2.4) chosen. For the order $l=2$ this possibility has been analyzed in detail for the two solutions (2.1-Ia) and (2.1-IIb). It is found that no non trivial extensions matching the ansatz (2.4) with $l=2$ are allowed. This lack of success for the ansatz (2.4) with $l=2$ suggests similar conclusions also hold for an ansatz with a higher value of $l$.

\section{Parametrization procedure for the elements of the SDRE}

In this section the principal formulae of the parametrization proposed in [7] and obtained by solving equations (1.4) will be summarized. In what follows, the dependence of spectral parameters and dynamical variables is implicit. When a quantity is non-dynamical it will be clearly stated. It is also assumed, as usual, that matrices $A, B, C, D$ are invertible.

First, the equation (1.4-c) together with the fact that $B$ is a space-1 zero weight matrix, allows to establish the existence of an invertible $(n \times n)$ matrix $b$ such that

$$
B_{12} \equiv C_{21}=b_{2}^{-1} b_{2}{ }^{g}\left(h_{1}\right) .
$$

This parametrization for the matrix $B$, by means of equation $(1.4-\mathbf{b})$, allows to prove the existence of a quasi non-dynamical $R$-matrix such that

$$
A_{12}=b_{1}^{-1}\left(b_{2}^{g}\right)^{-1} R_{12}\left(b_{1}\right)^{g} b_{2} .
$$

The $R$-matrix appearing in (3.2) is said to be quasi non-dynamical since the occurrence of the automorphism $g$ in (1.4-b) leads to the following constraint

$$
R_{12}=R_{12}{ }^{g g}\left(h_{3}\right) .
$$


As a consequence

$$
R_{12}=\left(e^{-\frac{\sigma}{\gamma}\left(\log g_{1}+\log g_{2}\right)}\right) R_{12}^{0}\left(e^{\frac{\sigma}{\gamma}\left(\log g_{1}+\log g_{2}\right)}\right), \quad \sigma=\sum_{k=1}^{n} \lambda_{k}
$$

where the matrix $R^{0}$, by means of equation (1.4-a), is proved to be a non-dynamical solution of the following $g$-deformed YBE

$$
R_{12}^{0} R_{13}^{0}{ }^{g g} R_{23}^{0}=R_{23}^{0}{ }^{g g} R_{13}^{0} R_{12}^{0}{ }^{g g} .
$$

Finally, the matrix $D$ is assumed to be decomposable as

$$
D_{12}=q_{1}^{-1}\left(h_{2}\right) q_{2}^{-1} \tilde{R}_{12} q_{1} q_{2}\left(h_{1}\right)
$$

where $\tilde{R}$ is also of the form (3.4). It should be pointed out that all constant $D$-matrices of weak Hecke type [12] associated to a Lie algebra $\mathbf{g}=g l(n)(n \geq 2)$ admit such a decomposition [13] with $g=\mathbb{1}$. This result was recently extended to the affine (trigonometric) dynamical $R$-matrices [14]. Such decompositions were already known in a number of cases (see for example [11] [16]). Indeed, they characterize the matrix $D$ as representation of a particular (cocycle) Drinfeld's twist [17] acting on a universal $R$-matrix, to yield a quasi-Hopf algebra structure. Note that one can show immediately the following [18]:

\section{Proposition 3.1}

If $R$ obeys the YBE (3.5), and $D$, which is constructed from $R$ as (3.4) and (3.6) for some $q$, is a zero-weight matrix, namely $\left[h_{i} \otimes \mathbb{1}+\mathbb{1} \otimes h_{i}, D_{12}\right]=0$ for $i=1, \ldots, n$, then $D$ obeys the GNF equation. In other words, the zero-weight condition is sufficient in the cocycle formulation (3.6).

Using parametrization (3.1)-(3.6) for structure matrices, one finds a consistent (sufficient) parametrization for the scalar solutions $K$ of the corresponding SDRE. In particular, one finds as a solution

$$
K=\left(b^{g}\right)^{-1} Q q
$$

where $Q$ solves

$$
R_{12} Q_{1} q_{1} Q_{2}\left(h_{1}\right) q_{1}^{-1}=Q_{2} q_{2} Q_{1}\left(h_{2}\right) q_{2}^{-1} \tilde{R}_{12}
$$

Since in all situations analyzed in the present article $K=\mathbb{1}$ is a solution of the SDRE, it is easily shown that one can choose $R=\tilde{R}$ and $q=b^{g}$ in (3.6). Therefore, consider the following parametrization for the matrix $D$

$$
D_{12}=\left(b_{1}^{g}\right)^{-1}\left(h_{2}\right)\left(b_{2}^{g}\right)^{-1} R_{12} b_{1}^{g} b_{2}^{g}\left(h_{1}\right) .
$$

If $Q$ is searched for as quasi non-dynamical, namely

$$
Q \equiv e^{-\frac{\sigma}{\gamma} \log g} Q^{0} e^{\frac{\sigma}{\gamma} \log g}
$$

with $Q^{0}$ non-dynamical, then (3.8) simplifies to the following modified YB-like equation 3

$$
R_{12}^{0} Q_{1}^{0} g_{2}^{-1} Q_{2}^{0} g_{2}=Q_{2}^{0} g_{1}^{-1} Q_{1}^{0} g_{1} R_{12}^{0}
$$

\footnotetext{
${ }^{3}$ Note that a more general situation is represented by replacing $g$ with $\tilde{g}$ in (3.10) and (3.11) provided $[g, \tilde{g}]=0$.
} 
This description emphasizes that a systematic scheme to build $A, B, C, D$ structure matrices arises. Indeed, starting from a given $D$-matrix, with a specific decomposition (3.6) yielding an $\tilde{R}$-matrix $(\tilde{R}=R)$ with a quasi non-dynamical property (3.3) such that the associated nondynamical matrix $R^{0}$ obeys a $g$-deformed YB equation (3.5), one has all the ingredients - namely $R, b$ and $g$ - to consistently build the remaining $A, B, C$ matrices.

At this stage it is worth recalling that non-scalar, operatorial solutions to the SDRE can also be obtained from (3.11) and (3.7). In fact, it is remarkably simple to prove the transfer matrix formula for the SDRE, such as obtained in [5], when it is expressed under a factorized form (see [7]). Indeed, it is possible to show the general 'dynamization of trace' as follows:

\section{Proposition 3.2}

Suppose $Q^{0}$ is a non-dynamical representation, for instance a monodromy matrix, of (3.11) for a given $R$-matrix $R^{0}$, and suppose $b$ is a dynamical matrix in $\operatorname{End}(\mathcal{U})$ such that $D_{12}=$ $b_{1}^{-1}\left(h_{2}\right) b_{2}^{-1} R_{12}^{0} b_{1} b_{2}\left(h_{1}\right)$ is zero-weight (we recall that the auxiliary space is $\mathcal{U}=V \otimes \mathbb{C}[u]$, and we take $g=\mathbb{1}$ for simplicity). It is then possible to construct a dynamical transfer matrix $\tau^{0}=\left(b^{-1} Q^{0} b e^{\partial_{\lambda}}\right)$ such that

$$
\left[\operatorname{Tr}_{V} \tau^{0}(u), \operatorname{Tr}_{V} \tau^{0}(v)\right]=0
$$

Proof. It is simple, provided the following technical tricks are used:

(a) for any three operators $\mathcal{M}_{1}, \mathcal{N}_{2}, \mathcal{O}_{2}$ not containing $e^{\partial_{\lambda}}$

$$
\operatorname{Tr}_{12}\left(\mathcal{M}_{1} e^{\partial_{1}} \mathcal{N}_{2} \mathcal{O}_{2} e^{\partial_{2}}\right)=\operatorname{Tr}_{12}\left(\mathcal{N}_{2}\left(h_{1}\right) \mathcal{M}_{1} e^{\partial_{1}} \mathcal{O}_{2} e^{\partial_{2}}\right)
$$

(b) for any zero-weight $\mathbb{C}$-number matrix $D_{12}$ and any operator $\mathcal{O}_{12}$ not containing $e^{\partial_{\lambda}}$

$$
\operatorname{Tr}_{12}\left(D_{12} \mathcal{O}_{12} D_{12}^{-1} e^{\left(\partial_{1}+\partial_{2}\right)}\right)=\operatorname{Tr}_{12}\left(\mathcal{O}_{12} e^{\left(\partial_{1}+\partial_{2}\right)}\right) 4
$$

Therefore, dynamical trace formula $\tau^{0}$ seems to be the one-space counterpart of the dynamical cocycle formula (3.6) .

\subsection{Parametrization for the ACF rational constant matrices}

The parametrization procedure will first be applied as an exercise to the structure matrices (1.10 1.12). In this case the auxiliary space $\mathcal{U}$ reduces to a finite dimensional vector space $V$ and $g=\mathbb{1}$.

Using expression (1.11) for $B$, a solution $b$ of (3.1) is

$$
b_{i j}^{\infty}=\prod_{1=a \neq j}^{n} \frac{\lambda_{j}^{(i-1)}}{\lambda_{j a}} i, j=1 \ldots n,
$$

where the notation $b^{\infty}$ emphasizes the independence from a spectral parameter. Note that such a solution is not unique. In fact, alternative solutions can be obtained by multiplying each row $i$ of the matrix (3.12) by a function $f_{i} \gamma$-periodic on each dynamical variable. Furthermore, it

\footnotetext{
${ }^{4}$ Two cases of this 'dynamical cyclicity' appear in [15]
} 
should be kept in mind that a matrix obtained by interchanging each pair of rows in (3.12) is still a solution of (3.1).

Similarly, a non-dynamical $R$-matrix solving (3.2) reads

$$
R^{\infty}=\sum_{i, j=1}^{n}\left(e_{i i} \otimes e_{j j}\right)+\gamma \sum_{i=1}^{n} \sum_{k=1}^{i-1} \sum_{j=1}^{i-k}\left(e_{i i-k} \otimes e_{j j+k-1}-e_{j j+k-1} \otimes e_{i i-k}\right),
$$

which is a Cremmer-Gervais $R$-matrix type [19]. Note that in this rational case there is a difference of one in the root heights, compared to the trigonometric case [13]. Clearly, in this case $R=R^{0} \equiv R^{\infty}$ and the matrix $R^{\infty}$ solves directly the ordinary YBE. The consequent explicit parametrization of the matrix $D^{\infty}(3.9)$ in terms of the non-dynamical $R^{\infty}$-matrix (3.13) and the 'twist' matrix $b^{\infty}(3.12)$ provides a concrete example of the theorem mentioned previously and proved in [13].

Finally, concerning the constant solutions $K$ of the SDRE, it can be verified that their corresponding $Q=Q^{\infty}$ matrices satisfy equation (3.10). Particularly, for solutions (2.1-IIa) and (2.1-IIb), these matrices are non dynamical and their expressions are particularly simple. For them, the $Q^{\infty}=Q^{0}$ matrices are

$$
\begin{array}{ll}
\text { IIa } & Q^{\infty}=\left(f e_{n n}+e_{n-11}+e_{n 2}\right) \gamma^{(n-1)}, \\
\text { IIb } & Q^{\infty}=\mathbb{1}+\sum_{i>j=1}^{n}\left(\begin{array}{c}
i-1 \\
i-j
\end{array}\right) f^{(i-j)} e_{i j} .
\end{array}
$$

As expected, (3.14-IIa) is a set of rank 2 matrices, while (3.14 IIIb) is an invertible triangular set of matrices. By contrast, solutions (2.1-Ia) and (2.1-Ib) cannot be de-dynamized by (3.7).

\subsection{Parametrization for the ACF rational non-constant matrices}

In this situation the structure matrices (1.7-1.9) are solutions of equation (1.4) with $g=$ $\exp \left(-\gamma \frac{d}{d u}\right)$. The parametrization presented formally in section (3) is still available, even if it appears to be a little more cumbersome. The 'twist' $b$ matrix is chosen as

$$
b_{i j}=\prod_{a \neq j} \frac{\lambda_{j}^{(i-1)}}{\lambda_{j a}}, \quad b_{n j}=\prod_{a \neq j} \frac{\lambda_{j}^{(n-1)}\left(\sigma-\lambda_{j}+u+\gamma\right)}{\lambda_{j a}\left(\sigma+u+f_{0}\right)} \quad j=1 \ldots n, \quad i=1 \ldots n-1,
$$

where $f_{0}$ is a function of $(\sigma+u)$. For simplicity, from now on, it will be taken to be a constant. Note that the spectral parameter dependence is limited to one single row of the matrix. Even 
this time this solution is not unique. Instead, the $R$-matrix reads

$$
\begin{aligned}
R= & \left(1+\frac{\gamma}{u-v}\right) \sum_{i=1}^{n-1} e_{i i} \otimes e_{i i}+\left(1+\frac{\gamma}{u-v}\right) \frac{\left(\sigma+v+f_{0}\right)\left(\sigma+u-\gamma+f_{0}\right)}{\left(\sigma+u+f_{0}\right)\left(\sigma+v-\gamma+f_{0}\right)} e_{n n} \otimes e_{n n} \\
& +\sum_{i j=1}^{n-1} e_{i i} \otimes e_{j j}+\frac{\left(\sigma+v+f_{0}\right)}{\left(\sigma+v-\gamma+f_{0}\right)} \sum_{i=1}^{n-1} e_{i i} \otimes e_{n n}+\frac{\left(\sigma+u-\gamma+f_{0}\right)}{\left(\sigma+u+f_{0}\right)} \sum_{i=1}^{n-1} e_{n n} \otimes e_{i i} \\
& +\gamma \frac{(\sigma+u+\gamma)}{\left(\sigma+u+f_{0}\right)} \sum_{k=1}^{n-1} \sum_{j=1}^{n-k} e_{i i-k} \otimes e_{j j+k-1}-\gamma \frac{(\sigma+v)}{\left(\sigma+v-\gamma+f_{0}\right)} \sum_{k=1}^{n-1} \sum_{j=1}^{n-k} e_{j j+k-1} \otimes e_{i i-k} \\
& +\gamma \sum_{i=1}^{n-1} \sum_{k=1}^{i-1} \sum_{j=1}^{i-k}\left(e_{i i-k} \otimes e_{j j+k-1}-e_{j j+k-1} \otimes e_{i i-k}\right)+\left(\frac{\gamma}{u-v}\right) \sum_{i \neq j=1}^{n-1} e_{i j} \otimes e_{j i} \\
& +\left(\frac{\gamma}{u-v}\right) \frac{\left(\sigma+u-\gamma+f_{0}\right)}{\left(\sigma+v-\gamma+f_{0}\right)} \sum_{i=1}^{n-1} e_{i n} \otimes e_{n i}+\left(\frac{\gamma}{u-v}\right) \frac{\left(\sigma+v+f_{0}\right)}{\left(\sigma+u+f_{0}\right)} \sum_{i=1}^{n-1} e_{n i} \otimes e_{i n} \\
& -\frac{\gamma}{\left(\sigma+u+f_{0}\right)} \sum_{i=1}^{n-1} \sum_{k=1}^{n-1-i} e_{n n-i} \otimes e_{k k+i}+\frac{\gamma}{\left(\sigma+v-\gamma+f_{0}\right)} \sum_{i=1}^{n-1} \sum_{k=1}^{n-1-i} e_{k k+i} \otimes e_{n n-i} .
\end{aligned}
$$

Note that the results obtained in the previous section are reproduced when the spectral parameters goes to infinity and consequently expressions (3.15) and (3.16) reduce to (3.12) and (3.13), respectively. As an example, for the specific case $n=2$ expression (3.16) becomes

$$
\begin{aligned}
R= & \left(1+\frac{\gamma}{u-v}\right)\left(e_{11} \otimes e_{11}+\frac{\left(\sigma+v+f_{0}\right)\left(\sigma+u-\gamma+f_{0}\right)}{\left(\sigma+v-\gamma+f_{0}\right)\left(\sigma+u+f_{0}\right)} e_{22} \otimes e_{22}\right) \\
& +\frac{\left(\sigma+v+f_{0}\right)}{\left(\sigma+v-\gamma+f_{0}\right)} e_{11} \otimes e_{22}+\frac{\left(\sigma+u-\gamma+f_{0}\right)}{\left(\sigma+u+f_{0}\right)} e_{22} \otimes e_{11} \\
& -\gamma \frac{(\sigma+v)}{\left(\sigma+v-\gamma+f_{0}\right)} e_{11} \otimes e_{21}+\gamma \frac{(\sigma+u+\gamma)}{\left(\sigma+u+f_{0}\right)} e_{21} \otimes e_{11} \\
& +\left(\frac{\gamma}{u-v}\right)\left(\frac{\sigma+u-\gamma+f_{0}}{\sigma+v-\gamma+f_{0}} e_{12} \otimes e_{21}+\frac{\sigma+v+f_{0}}{\sigma+u+f_{0}} e_{21} \otimes e_{12}\right) .
\end{aligned}
$$

Notice that the choice $f_{0}=\gamma$ enables to simplify a little this expression for the $R$-matrix.

As expected, and unlike the previous case, the $R$-matrix is still dynamical. However, in agreement with (3.4), the dynamical dependence can indeed be eliminated and, setting $f_{0}=\gamma$, the 
matrix $R^{0}$ reads

$$
\begin{aligned}
R^{0}= & \left(1+\frac{\gamma}{u-v}\right) \sum_{i=1}^{n-1} e_{i i} \otimes e_{i i}+\left(1+\frac{\gamma}{u-v}\right) \frac{u(v+\gamma)}{v(u+\gamma)} e_{n n} \otimes e_{n n} \\
& +\sum_{i j=1}^{n-1} e_{i i} \otimes e_{j j}+\frac{(v+\gamma)}{v} \sum_{i=1}^{n-1} e_{i i} \otimes e_{n n}+\frac{u}{(u+\gamma)} \sum_{i=1}^{n-1} e_{n n} \otimes e_{i i} \\
& +\gamma \sum_{i=1}^{n} \sum_{k=1}^{i-1} \sum_{j=1}^{i-k}\left(e_{i i-k} \otimes e_{j j+k-1}-e_{j j+k-1} \otimes e_{i i-k}\right)+\left(\frac{\gamma}{u-v}\right) \sum_{i \neq j=1}^{n-1} e_{i j} \otimes e_{j i} \\
& +\left(\frac{\gamma}{u-v}\right) \frac{u}{v} \sum_{i=1}^{n-1} e_{i n} \otimes e_{n i}+\left(\frac{\gamma}{u-v}\right) \frac{(v+\gamma)}{(u+\gamma)} \sum_{i=1}^{n-1} e_{n i} \otimes e_{i n} \\
& -\frac{\gamma}{(u+\gamma)} \sum_{i=1}^{n-1} \sum_{k=1}^{n-1-i} e_{n n-i} \otimes e_{k k+i}+\frac{\gamma}{v} \sum_{i=1}^{n-1} \sum_{k=1}^{n-1-i} e_{k k+i} \otimes e_{n n-i} .
\end{aligned}
$$

It should be noticed that this $R$-matrix does depend on the two spectral parameters $u$ and $v$ independently and not only through their difference. This represents a novelty with respect to the $R$-matrices which are solutions of the standard YBE, which usually depend on the spectral parameters only through their difference. Using (3.15) and (3.16), a parametrization for the $D$-matrix in line with (3.9) is realized. It represents an interesting example of decomposition for non constant $D$-matrices.

Again, the matrix $Q$ (3.7) for the set of solutions (2.14) can be calculated. For instance, the $Q$ matrices for the invertible solutions (2.14-IIb), which do depend on the dynamical variables, are given by the following triangular matrices

IIb

$$
Q=\sum_{i=1}^{(n-1)} e_{i i}+\left(1-\frac{n f}{u}\right) e_{n n}+\sum_{i>j=1}^{(n-1)}\left(\begin{array}{c}
i-1 \\
i-j
\end{array}\right) f^{(i-j)} e_{i j}+\frac{M_{n j}}{u\left(u+\sigma-\gamma+f_{0}\right)} e_{n j},
$$

with

$$
M_{n j}=\sum_{j=1}^{(n-1)}\left(\begin{array}{c}
n-1 \\
n-j
\end{array}\right) f^{(n-j)} u(u+\sigma)-\left(\begin{array}{c}
n \\
n-j+1
\end{array}\right) f^{(n-j+1)} u+(-)^{(n-j+1)} n f \sum_{\beta \in \mathcal{L}_{n j}} \prod_{i \in \beta} \lambda_{i},
$$

where $\mathcal{L}_{n j}$ is a set of set of indexes depending on $n$ and $j$ : elements $\beta$ of $\mathcal{L}_{n j}$ are all distinct sets of $(n-j+1)$ different indexes $k=1, \ldots, n$. It can be easily seen that (3.19) collapses to (3.14) in the limit $u \longrightarrow \infty$. This expression however does not allow for a factorization of the dynamical shift in equation (3.8), which, therefore, cannot be simplified to the form (3.11). 


\section{New set of structure matrices for the SDRE}

The work by Antonov et al. [11] provides an alternative parametrization for the matrix ${ }^{\text {trig }} D$ corresponding to the trigonometric case for which expression (1.9) is the rational limit.5 This fact suggests the possibility to use such a decomposition for finding new solutions for the matrices $A, B$ and $C$ satisfying the consistency equations (1.4). More precisely, the parametrization provided in [11] concerns the matrix ${ }^{t r i g} D^{T}$ and can be written as follows

$$
S_{12} \tilde{c}_{1} \tilde{c}_{2}\left(\lambda-\gamma h_{1}\right)=\tilde{c}_{2} \tilde{c}_{1}\left(\lambda-\gamma h_{2}\right)^{t r i g} D_{12}^{T} ; \quad S_{12}=d_{1}^{-1} \tilde{S}_{12} d_{2}
$$

with

$$
\begin{aligned}
& \quad \sum_{i \neq j=1}{ }^{\text {trig }} D_{12}\left[\frac{\sinh (s+\gamma)}{\sinh (s)} e_{i i} \otimes e_{i i}+\frac{\sinh (\gamma) \sinh \left(s+\lambda_{i j}\right)}{\sinh (s) \sinh \left(\lambda_{i j}\right)} e_{i j} \otimes e_{j i}+\frac{\sinh \left(\lambda_{i j}-\gamma\right)}{\sinh \left(\lambda_{i j}\right)} e_{i i} \otimes e_{j j}\right] \\
& \quad S_{12} \\
& =\sum_{i \neq j=1}^{n}\left[\frac{\sinh (s+\gamma)}{\sinh (s)} e_{i i} \otimes e_{i i}+\frac{\sinh (\gamma)}{\sinh (s)} e^{s(2(i-j)-n \operatorname{sign}(i-j)) / n} e_{i j} \otimes e_{j i}+e^{\gamma \operatorname{sign}(i-j)} e_{i i} \otimes e_{j j}\right] \\
& +2 \sinh (\gamma)\left[\sum_{1=i<i^{\prime}<j}^{n} e^{-2 s\left(i^{\prime}-i\right) / n} e_{i i^{\prime}} \otimes e_{j j^{\prime}}-\sum_{i>i^{\prime}>j=1}^{n} e^{-2 s\left(i^{\prime}-i\right) / n} e_{i i^{\prime}} \otimes e_{j j^{\prime}}\right] i+j=i^{\prime}+j^{\prime},
\end{aligned}
$$

where $s=(u-v)$ and the elements of the matrices $c$ and $d$ are

$$
\tilde{c}_{j k}=e^{2 j\left(u+n \lambda_{k}\right) / n}, \quad d_{j k}=e^{2 j \gamma / n} \delta_{j k} .
$$

The notation adopted in writing these formulas has been adapted to the present article, and therefore it differs slightly from the conventions used in [11]. The matrix $\tilde{S}$ from [11] is nondynamical and depends on the spectral parameters only through their difference $s$. According to Antonov et al. it satisfies the YBE, and consequently, the matrix $S$ satisfies the YBE as well, since the following property holds

$$
[S, d \otimes d]=0 .
$$

As it is, the expression (4.1) implies a parametrization for the ${ }^{\text {trig }} D$-matrix which does not match the decomposition (3.6). However, it can be noticed that both matrices $\tilde{S}$ and ${ }^{\text {trig }} D$ are invariant under the following transformation

$$
\text { space } 1 \longleftrightarrow \text { space } 2, \quad \gamma \longrightarrow-\gamma \text {. }
$$

\footnotetext{
${ }^{5}$ Note that recently, all these parametrizations received a universal description in [14].
} 
This fact allows to rewrite expression (4.1) as follows

$$
S_{12} \tilde{c}_{2}, \tilde{c}_{1}\left(\lambda+\gamma h_{2}\right)=\tilde{c}_{1} \tilde{c}_{2}\left(\lambda+\gamma h_{1}\right)^{\text {trig }} D_{12}^{T},
$$

and consequently to obtain a parametrization for ${ }^{t r i g} D$ in the form (3.6), namely

$$
S_{12}^{T} c_{1} c_{2}\left(\lambda+\gamma h_{1}\right)=c_{2} c_{1}\left(\lambda+\gamma h_{2}\right)^{\text {trig }} D_{12} ; \quad c_{j}=\left(\tilde{c}_{j}^{-1}\right)^{T} \quad j=1,2 .
$$

It can be noticed that unlike the case investigated previously in section (3.2), the automorphism $g$ is set equal to $\mathbb{1}$, in spite of a spectral parameter dependence. At this stage, putting in effect the procedure sketched in section (3), it is possible to derive new matrices $A, B$ and $C$ from the new set of data associated to ${ }^{\text {trig }} D$, namely $S^{T}=R, c=b$ and $g=\mathbb{1}$. For instance, one can think to obtain immediately the rational limit of the matrices $c$ and $S^{T}$, hence suitable formulations for matrices $b$ and $R$ (3.9), and consequently to find the corresponding matrices $B$ and $A$ using expressions (3.1) and (3.2), respectively. Unfortunately, the rational limit of matrix $c$, unlike the trigonometric case, leads to a matrix which is non-invertible. An alternative possibility is first to find matrices $B$ and $A$ in the trigonometric case, and subsequently to take their well-defined, non-singular rational limit consistently with equations (1.4). This last procedure turns out to be a better strategy. The new rational matrices $B$ and $A$, which will be indicated as $\hat{B}$ and $\hat{A}$ to differentiate them from the ACF matrices, are:

$$
\begin{aligned}
\hat{B}_{12}= & \sum_{i=1}^{n} e_{i i} \otimes p_{i}, \\
p_{i}= & \prod_{1=k \neq i}^{n} \frac{\lambda_{i k}}{\left(\lambda_{i k}+\gamma\right)} e_{i i}+\sum_{1=j \neq i}^{n}\left(e_{j j}-\gamma \prod_{1=k \neq i, j}^{n} \frac{\lambda_{i k}}{\lambda_{j k}\left(\lambda_{j i}-\gamma\right)} e_{i j}\right) \\
\hat{A}_{12}= & \sum_{i=1}^{n}\left(1+\frac{\gamma}{s}\right) e_{i i} \otimes e_{i i}+\sum_{i \neq j=1}^{n}\left[\left(1-\frac{\gamma}{\lambda_{i j}}\right) e_{i i} \otimes e_{j j}+\left(\frac{\gamma}{s}+\frac{\gamma}{\lambda_{i j}}\right) e_{i j} \otimes e_{j i}\right] \\
& +\sum_{i \neq j=1}^{n} \prod_{k \neq i, j ; l \neq j}^{n} \frac{\gamma \lambda_{i k}}{\lambda_{j l}}\left(e_{i i} \otimes e_{i j}-e_{i j} \otimes e_{i i}\right), \quad s=(u-v) .
\end{aligned}
$$

Since matrix $B$ - called $\hat{B}$ in the present case - is known, expression (3.1) can be used to find a suitable invertible $b$ matrix, which, in the present case, turns out to be given by the following expression

$$
\hat{b}_{i j}=\frac{\sum_{\alpha \in \mathcal{I}_{i j}} \prod_{l \in \alpha} \lambda_{l}}{\prod_{k \neq j}^{n} \lambda_{j k}},
$$

where $\mathcal{I}_{i j}$ is a set depending on $i$ and $j$. Each element $\alpha$ of $\mathcal{I}_{i j}$ is a collection of $(n-i)$ different indexes $l \neq j$ and the total number of elements of this set is given by the binomial coefficient $(n-1) ! /(n-i) !(i-1)$ !. For instance, for $n=3$ (4.11) becomes is:

$$
\hat{b}=\left(\begin{array}{ccc}
\frac{\lambda_{2} \lambda_{3}}{\lambda_{12} \lambda_{13}} & \frac{\lambda_{1} \lambda_{3}}{\lambda_{21} \lambda_{23}} & \frac{\lambda_{1} \lambda_{2}}{\lambda_{31} \lambda_{32}} \\
\frac{\lambda_{2}+\lambda_{3}}{\lambda_{12} \lambda_{13}} & \frac{\lambda_{1}+\lambda_{3}}{\lambda_{21} \lambda_{23}} & \frac{\lambda_{1}+\lambda_{2}}{\lambda_{31} \lambda_{32}} \\
\frac{1}{\lambda_{12} \lambda_{13}} & \frac{1}{\lambda_{21} \lambda_{23}} & \frac{1}{\lambda_{31} \lambda_{32}}
\end{array}\right) .
$$


Since matrices $\hat{b}$ and $\hat{A}$ are available, relation (3.2) can be used for computing the $R$-matrix which satisfies the ordinary YBE and which is

$$
\begin{aligned}
\hat{R}_{12}= & \sum_{i=1}^{n}\left(1+\frac{\gamma}{s}\right) e_{i i} \otimes e_{i i}+\sum_{i \neq j=1}^{n}\left(e_{i i} \otimes e_{j j}+\frac{\gamma}{s} e_{i j} \otimes e_{j i}\right) \\
& +\gamma \sum_{i=1}^{n} \sum_{k=1}^{i-1} \sum_{j=1}^{i-k}\left(e_{i-k i} \otimes e_{j+k-1 j}-e_{j+k-1 j} \otimes e_{i-k i}\right) .
\end{aligned}
$$

As expected, this matrix is non-dynamical. In addition it depends on the spectral parameters only through their difference $s$. It can be noticed that in the limit without spectral parameter, expression (4.13) becomes the transposed of matrix (3.13). Once again the rational CremmerGervais-type matrix $\hat{R}$ exhibits a difference of one in root heights, compared to the trigonometric case [14]. This fact also explains why one cannot take the direct trigonometric to rational limit in this procedure, since the underlying non-dynamical matrices are definitively of distinct form.

None can be said about the parametrization of the solutions $K$ of the SDRE with the structure matrices presented in this section, since no $K$ matrices are known yet. However, it is interesting to see whether the parametrization procedure could provide some information concerning these unknown solutions and act as a shortcut for finding them. Consider the situation with constant ACF structure matrices, for which the 'twist' is constructed from (3.12), and consider also the parametrization for $D$ (3.6) with the 'twist' built from (4.11). Then, (3.8) becomes

$$
R_{12} Q_{1} \hat{b}_{1} Q_{2}\left(h_{1}\right) \hat{b}_{1}^{-1}=Q_{2} \hat{b}_{2} Q_{1}\left(h_{2}\right) \hat{b}_{2}^{-1} \hat{R}_{12} \quad Q=b K \hat{b}^{-1} .
$$

All elements of this expression are known since the $K$ matrices refer to solutions of the SDRE with ACF structure matrices. The same cannot be said concerning the following expression

$$
\hat{R}_{12} \hat{Q}_{1} b_{1} \hat{Q}_{2}\left(h_{1}\right) b_{1}^{-1}=\hat{Q}_{2} b_{2} \hat{Q}_{1}\left(h_{2}\right) b_{2}^{-1} R_{12}, \quad \hat{Q}=\hat{b} \hat{K} b^{-1},
$$

which is obtained from the SDRE using the new set of constant structure matrices, for which the 'twist' is constructed from (4.11) and the parametrization for the $D$-matrix is obtained using the 'twist' built from (3.12). In this case the matrices $\hat{K}$ are unknown. However, (4.14) can be manipulated in such a way to end up matching the formulation (4.15). Writing (4.14) as

$$
\hat{R}_{12}\left[Q_{1} \hat{b}_{1} Q_{2}\left(h_{1}\right) \hat{b}_{1}^{-1}\right]^{-1}=\left[Q_{2} \hat{b}_{2} Q_{1}\left(h_{2}\right) \hat{b}_{2}^{-1}\right]^{-1} R_{12},
$$

it can be verified that (4.15) and (4.16) coincide provided

$$
Q_{2}^{-1}\left(h_{1}\right)\left(\hat{b}_{1}^{-1} Q_{1}^{-1} b_{1}\right)=\left(\hat{b}_{1}^{-1} \hat{Q}_{1} b_{1}\right) \hat{Q}_{2}\left(h_{1}\right),
$$

and therefore

$$
\left(\hat{b}_{2} K_{2}^{-1} b_{2}^{-1}\right)\left(h_{1}\right) K_{1}^{-1}=\hat{K}_{1}\left(\hat{b}_{2} \hat{K}_{2} b_{2}^{-1}\right)\left(h_{1}\right),
$$

which represents a relation amongst the invertible constant solutions $K$ and $\hat{K}$ of the SDRE with the two different sets of structure matrices. Full investigation of this equation will be left to future studies, however something can be said immediately concerning the simplest case, namely $n=2$. In fact, making use of the corresponding invertible set of solutions $K$ (2.1-IIb), it is possible to compute

$$
Q=\left(\begin{array}{rc}
0 & 1 \\
-1 & f+\sigma
\end{array}\right)
$$


Though (4.19) is dynamical, its dependence from the dynamical variables appears through $\sigma$. This fact allows to simplify (4.18) which now reads

$$
K_{1}^{-1}\left(\hat{b}_{2} K_{2}^{-1} b_{2}^{-1}\right)(\sigma+\gamma)=\hat{K}_{1}\left(\hat{b}_{2} \hat{K}_{2} b_{2}^{-1}\right)\left(h_{1}\right) .
$$

As a consequence

$$
\hat{K}=K^{-1}=\left(\begin{array}{cc}
1-\frac{f}{\lambda_{12}} & \frac{f}{\lambda_{12}} \\
-\frac{f}{\lambda_{12}} & 1+\frac{f}{\lambda_{12}}
\end{array}\right),
$$

represents a set of invertible constant solutions of the SDRE for the new set of constant matrices proposed in this section in the case $n=2$. In the limit $f \longrightarrow 0$, the solution $\hat{K}=\mathbb{1}$ is obtained. More generally, when the mixed matrices $Q$ (4.14) built from the 2 cocycles $b$ and $\hat{b}$ are quasi non-dynamical, $\hat{K}=K^{-1}$ always provides a solution to the alternative SDRE. Unfortunately, in the present case, for $n>2$ the dynamical dependence of matrices $Q$ cannot be formulated in terms of $\sigma$ and therefore a more careful investigation of equation (4.18) is needed for finding matrices $\hat{K}$.

\section{Conclusion}

The purpose of this article has been to extend previous work concerning the classification of constant solutions of the SDRE to the non-constant case, and to provide explicit realizations of the parametrization procedure proposed in [7] for all elements of the SDRE. During this analysis it has been shown how the existence of two distinct parametrizations for the $D$-matrix leads to different sets of structure matrices for the SDRE, and consequently to new solutions $K$ for this equation. Because of the parametrization procedure, it was possible to reveal a connection amongst invertible solutions $K$ of the SDRE equation with the two different sets of structure matrices available. In this context, an explicit example has been provided for the case $n=2$. Still, a full investigation of the SDRE equation is required for obtaining a classification of the solutions $K$ related to the new set of structure matrices. It will be interesting to see the relationship amongst the integrable systems stemming from these solutions and the RS models related to the ACF matrices, since all of them share the same $D$-matrix. In addition, the existence, exemplified here, of several inequivalent sets of $A, B, C, D$ matrices, which share the same $D$-matrix with different de-twisting procedures (3.6), may explain why only two sets out of four sets of constant solutions $K^{\infty}$ (2.1) can be extended to the non-constant case (2.14). In fact, besides the ACF set of structure matrices $A, B, C, D$ used in the present article, there may exist another set $A^{\prime}, B^{\prime}, C^{\prime}, D$ with the same limit $u, v,(u-v) \longrightarrow \infty$ and different non-constant solutions $K^{\prime}$, this time for the other two sets of $K^{\infty}$. The new set $\hat{A}, \hat{B}, \hat{C}, D$ found in section (4) does not realized this scheme since $\hat{R}^{\infty}, \hat{b}^{\infty}$ are different from $R^{\infty}, b^{\infty}$.

Key objects of the parametrization procedure are 'twists' and $R$-matrices for which explicit formulations are provided. Amongst the $R$-matrices found, it is worth pointing out matrices (3.18), which satisfy a shifted YBE and which seem to appear here for the first time. Existence of two decompositions of the matrix $D$ does not contradict the uniqueness theorems in [13, 14] since the decomposition (3.6) does not yield an $R$-matrix solving the Yang-Baxter equation but its shifted extension. 
Building explicit monodromy matrices and consequently $N$-body system or spin-chain Hamiltonians is now feasible. Once again, it should be emphasized that in [7] it was shown how the parametrization procedure proposed is able to extremely simplify these constructions providing an elegant factorized form for the monodromy matrices. This is due to the possibility to eliminate completely the quantum-space shifts of the dynamical variables, which are present in the original formulas [6], and which make the construction of suitable monodromy matrices particularly cumbersome.

Finally, it should be emphasized that the ACF matrices satisfying the SDRE are associated to RS models in the bulk. In fact, the SDRE is not a reflection equation in the usual sense and in [4] it was shown how any representation of the algebra (1.1) with the ACF structure matrices turns into a representation of the fundamental relation $S L L=L L S$ provided suitable transformations are applied. This fact can be also seen as a rational and general consequence of the parametrization procedure presented in section (3) and applied to the ACF structure matrices. It would be interesting to study the RS models with a boundary and to find a suitable algebra able to describe them.

\section{Acknowledgements}

C.Z. thanks the Centre National de la Recherche Scientifique (CNRS) for the postdoctoral fellowship SPM 06-13, and J.A. thanks Universidad do Algarve for their hospitality.

\section{References}

[1] E. K. Sklyanin, Boundary conditions for integrable quantum systems, J. Phys. A21 (1988), 2375 .

[2] I. Cherednik, Factorizing particles on a half-line and root systems, Theor. Math. Phys. 61 (1984), 977;

L. Freidel and J. M. Maillet, Quadratic algebras and integrable systems, Phys. Lett. B262 (1991), 278;

P. P. Kulish and E. K. Sklyanin, The general $U_{q}[s l(2)]$ invariant $X X Z$ integrable quantum spin chain, J. Phys. A24, (1992), L435.

[3] H. Fan, B. Y. Hou and K. J. Shi, Integrable $A_{n-1}^{1.1}$ IRF model with reflecting boundary conditions, Mod. Phys. Lett. A26 (1997), 1929;

J. Donin and A. I. Mudrov, Reflection equation, twist and equivalent quantization; math-qa/0204295;

J. Donin, P. P. Kulish and A. I. Mudrov, On universal solutions to reflection equation, Lett. Math. Phys. 63 (2003), 179; math-qa/0210242;

P. P. Kulish and A. I. Mudrov, Dynamical reflection equation; math-qa/0405556.

[4] G. E. Arutyunov, L. O. Chekhov and S. A. Frolov, R-matrix quantization of the elliptic Ruijsenaar-Schneider model, Comm. Math. Phys. 192 (1998), 405; q-alg/9612032. 
[5] J. Avan, G. Rollet and Z. Nagy, Construction of dynamical quadratic algebras, Lett. Math. Phys. 67 (2004), 1.

[6] J. Avan and Z. Nagy, Spin chains from dynamical quadratic algebras, J. Stat. Mech. 2 (2005) P03005; math.QA/050129.

[7] J. Avan, and G. Rollet, Parametrization of semi-dynamical quantum reflection algebra, J. Phys. A40 (2007) 2709; math.QA/0611184v2.

[8] J. Avan, and G. Rollet,Classification of the solutions of constant rational semi-dynamical reflection equations; Ann. Henri Poincaré 7 (2006) 1463; nlin.SI/0511067.

[9] J. L. Gervais and A. Neveu, Novel triangle relations and the absence of tachyons in Liouville string field theory, Nucl. Phys. B238 (1984), 125;

G. Felder, Conformal field theory and integrable systems associated to elliptic curves, Proceedings ICM Zurich (1994), 1247, Proceedings ICMP Paris (1994), 211; hep-th/9407154.

[10] S. N. M. Ruijsenaars and H. Schneider, A new class of integrable systems and its relation to solitons, Ann. Phys. 170 (1986), 370;

S. N. M. Ruijsenaars, Complete integrability of relativistic Calogero-Moser systems, Comm. Math. Phys. 110 (1987), 191.

[11] A. Antonov, K. Hasegawa and A. Zabrodin, On trigonometric interwining vectors and non-dynamical R-matrix for the Ruijsenaars model, Nucl. Phys. B503 (1997), 747; hep.th/9704074.

[12] L. D. Faddeev, N. Yu. Reshetikhin and L. A. Takhtajan, Quantization of Lie groups and Lie algebras, Leningrad Math. J. 1 (1990), 193.

[13] E. Buffenoir, P. Roche and V. Terras, Quantum dynamical coboundary equation for finite dimensional simple Lie algebras, Adv. Math. 214 (2007), 181; math.QA/0512500.

[14] E. Buffenoir, P. Roche and V. Terras, Universal vertex-IRF transformation for quantum affine algebras; arXiv:0707.0955v1 [math-ph].

[15] J. Avan, Z. Nagy, A. Doikou and G. Rollet, Commuting quantum traces for quantum algebras, J. Math. Phys. 46 (2005), 083516; math/0403246 [math.QA].

[16] A. Arnaudon, E. Buffenoir, E. Ragoucy and Ph. Roche, Universal solutions of quantum Dynamical Yang-Baxter equations, Lett. Math. Phys. 44 (1998), 201;

M. Jimbo, H. Konno, S. Odake and J. Shiraishi, Quasi-Hopf twistors for elliptic quantum groups, Transform. Groups 4 (1999), 302

[17] V. G. Drinfeld, Quasi-Hopf algebras, Leningrad Math. Journ. 1 (1990), 1419.

[18] O. Babelon, private communication.

[19] E. Cremmer and J.L. Gervais, The quantum group structure associated with non-linearly extended Virasoro algebras, Comm. Math. Phys. 134 (1990), 619. 